\title{
Desafios para regionalização da Vigilância em Saúde na percepção de gestores de regiões de saúde no Brasil
}

\author{
Challenges for regionalizing surveillance in the perception of \\ managers of health regions in Brazil
}

\author{
Ana Coelho de Albuquerque $\mathbf{1}^{\mathbf{1}}$ Eduarda Ângela Pessoa Cesse ${ }^{\mathbf{2}}$, Isabella Chagas Samico', Eronildo \\ Felisberto', Paulo Germano de Frias', Geison Cícero da Silva1
}

DOI: 10.1590/0103-1104202112802

RESUMO O objetivo deste estudo foi descrever a percepção de gestores sobre os desafios da regionalização da Vigilância em Saúde em seis regiões de saúde no Brasil. Adotaram-se abordagem qualitativa e roteiro de entrevista semiestruturado, elaborado a partir de categorias de análise previamente definidas, aplicado a seis gestores estaduais da vigilância. Identificaram-se convergências e divergências entre regiões a partir das narrativas, examinadas por meio da técnica de análise temática de conteúdo. Constatou-se que a regionalização da vigilância é influenciada pelo contexto local, caminha de forma assimétrica, com o protagonismo dos estados, porém com escassas estratégias de fortalecimento. Entre os desafios, observaram-se dificuldades na apropriação de espaços de pactuação e cogestão e pouca participação da Vigilância em Saúde nas pautas de discussão da Comissão Intergestores Regional; na integração das ações da vigilância e atenção básica; na atuação da vigilância nas redes de atenção à saúde; e inexistência de normativas que regulem o financiamento regional. Ainda que as convergências identificadas indiquem um caminho para implementação da regionalização da Vigilância em Saúde, as contradições entre o legitimado como desejável e o instituído como prática representam limites concretos de efetivação da regionalização.

PALAVRAS-CHAVE Vigilância em saúde pública. Regionalização. Políticas. Política de saúde.

1 Instituto de Medicina Integral Prof. Fernando Figueira (Imip), Grupo de Estudos em Gestão e Avaliação em Saúde (Geas) - Recife (PE), Brasil. ana_albuquerque1@hotmail. com

2 Fundação Oswaldo Cruz (Fiocruz), Instituto Aggeu Magalhães (IAM) - Recife (PE), Brasil.

\begin{abstract}
The article aims to describe the perception of managers regarding the challenges of regionalization of Health Surveillance (HS) in six health regions in Brazil. A qualitative exploratory approach was adopted with data from semi-structured interviews, with a road map elaborated from previously defined categories of analysis, applied to six state managers of surveillance. Convergences and divergences were identified between regions based on the interviewees' discourses, which were examined through the thematic content analysis technique. The results showed that the regionalization of surveillance is influenced by the local context, its course is asymmetrical, with the states leading the process, but with few strengthening strategies. Among the challenges for its consolidation, there were difficulties in the appropriation of spaces for co-ordination and co-management, with little participation of $H S$ in the discussion guidelines of the Regional Interagency Committee; in the integration of surveillance and basic care actions; in the performance of surveillance in health care networks; in addition to the lack of regulations ruling regional financing. Although the identified convergences indicate a way to implement the regionalization of $H S$, the contradictions between what is legitimized as desirable and what is established as practice represent concrete limits to the accomplishment of regionalization.
\end{abstract}

KEYWORDS Public health surveillance. Regional health planning. Policy. Health policy. 


\section{Introdução}

A Lei Orgânica da Saúde integra o arcabouço normativo do Sistema Único de Saúde (SUS), trata dos seus princípios e diretrizes e referenda a regionalização como parte do processo de descentralização político-administrativa do sistema'. No entanto, há tensionamentos quanto às atribuições e às competências do Ministério da Saúde (MS), das secretarias estaduais e municipais de saúde no processo de implementação da regionalização ${ }^{2}$, decorrente do 'federalismo sanitário brasileiro' e sua configuração em gestão, organização e funcionamento espelharem o sistema federativo nacional ${ }^{3}$.

A política de saúde no Brasil desenvolveu mecanismos de indução e coordenação, que acomodaram as tensões federativas nos processos de descentralização e regionalização do SUS ${ }^{4,5}$. A regulação realizada pelo MS baseada na edição de portarias, em geral associadas a mecanismos financeiros, favoreceu a adesão e a implementação das políticas pelos gestores locais e estaduais ${ }^{6}$, bem como o aprendizado institucional das secretarias de saúde ${ }^{7}$. Ainda assim, a maioria dos municípios e muitos estados não têm condições para prover autonomamente as ações e serviços necessários à sua população, sendo indispensável transcender suas fronteiras sem, contudo, desconsiderá-las, para planejar e conformar uma rede de atenção integral à saúde no território ${ }^{8-10}$.

Nesse sentido, o MS, o Conselho Nacional de Secretários de Saúde (Conass) e o Conselho Nacional de Secretarias Municipais de Saúde (Conasems) pactuaram, na Comissão Intergestores Tripartite (CIT), as diretrizes e os critérios para a regionalização e o planejamento regional integrado do SUS, considerando que o seu fortalecimento promove a equidade, a integralidade na atenção à saúde, a racionalização dos gastos e a otimização dos recursos. De acordo com as resoluções, uma vez definidas as prioridades sanitárias regionais, é necessário pactuar as responsabilidades individuais e solidárias de cada ente federado no âmbito regional, abrangendo ações de assistência à saúde, de promoção, de assistência farmacêutica e de Vigilância em Saúde (VS), a serem realizadas no território ${ }^{\mathbf{1 1}, 12}$.

A legislação brasileira vigente compreende a VS como um processo sistemático que envolve desde a coleta e análise até a disseminação de dados sobre eventos relacionados com a saúde, articulando saberes e práticas relativas às vigilâncias: epidemiológica, ambiental, do trabalhador e sanitária, com vistas à implementação de políticas públicas de promoção da saúde, proteção, prevenção e controle de riscos, agravos e doenças ${ }^{13}$. Porém, estabelecer os limites entre as ações locais e as que necessitam de articulação regional relacionadas com a VS permanece como desafio, em particular, pela ausência de instruções normativas sobre o compartilhamento de atribuições, de recursos humanos, de materiais, além do financiamento regional ${ }^{14}$, sugerindo um insuficiente acúmulo gerencial da VS para esse nível organizacional ${ }^{15}$.

Esse contexto mostra a organização da VS no Brasil com dimensões claramente instituídas, representada por sistemas de normas, história e valores que orientam as organizações, enquanto outra dimensão instituinte tensiona ao mostrar necessidade de incorporar transformações nas concepções e modo de operar tradicionalmente e nas relações de poder onde esteja agindo ${ }^{2,16}$.

A municipalização priorizada e induzida pela instância nacional, com a criação de mecanismos estáveis de financiamento por meio da transferência fundo a fundo federal para o nível municipal17, e as iniciativas de qualificação das ações locais, como o Projeto Vigisus $^{18}$ e o Programa de Qualificação das Ações de Vigilância em Saúde (PQAVS) ${ }^{19}$, secundarizaram o processo de regionalização. Ainda assim, por meio de estímulos técnico-políticos, a regionalização do SUS vem sendo construída por gestores estaduais e municipais influenciados por ciclos de implementação das políticas e pelo grau de articulação/cooperação existente entre os atores sociais que compõem os espaços de governança do setor ${ }^{20}$. 
É possível que esse contexto institucional interfira na regionalização da VS no Brasil, tensionando a relação entre o normatizado e o que é operacionalizado nas regiões de saúde. Assim, este artigo tem como objetivo descrever a percepção de gestores com relação aos desafios para implementação da regionalização da VS em seis regiões de saúde no Brasil.

\section{Material e métodos}

Este estudo é um recorte de pesquisa maior sobre Regiões e Redes de Atenção à Saúde no Brasil21, em que o público-alvo foi composto por gestores estaduais de VS. Devido à natureza do objeto investigado, o método escolhido foi qualitativo, o qual utilizou entrevista semiestruturada como técnica de coleta das informações. Por meio da narrativa dos sujeitos, é possível apreender as percepções sobre os desafios para a implementação da regionalização da vigilância.

Para compor a amostra de sujeitos, partiu-se da seleção das regiões de saúde baseada em tipologia desenvolvida por Viana et al.22, que adotaram a análise de cluster para desenvolvimento socioeconômico e a oferta e complexidade dos serviços de saúde no contexto regional como condições estruturais de regionalização. Foram diferenciados cinco agrupamentos de regiões de saúde: 1) baixo desenvolvimento socioeconômico e baixa oferta de serviços; 2) médio/alto desenvolvimento socioeconômico e baixa oferta; 3) médio desenvolvimento socioeconômico e média oferta de serviços; 4) alto desenvolvimento socioeconômico e média oferta de serviços; e 5) alto desenvolvimento socioeconômico e alta oferta de serviços.

Selecionou-se uma região de cada grupo, além de outra interestadual por sua representatividade ímpar, de unidades federadas distintas, totalizando seis regiões: $\mathrm{V}$ Diretoria Regional de Saúde do estado de São Paulo; Região de Saúde do entorno de Manaus e Alto Rio Negro, Amazonas; Região de Saúde da Baixada Cuiabana, Mato Grosso; Região de Saúde 9 - Carbonífera/Costa Doce, Rio Grande do Sul; VIII Região de Saúde de Pernambuco; e Núcleo Regional de Saúde Norte, Bahia. A partir dessas regiões, identificaram-se os gestores estaduais da VS como condutores políticos e técnicos da regionalização, os quais foram contatados previamente pela equipe de pesquisadores e entrevistados nos seus locais de trabalho entre agosto de 2015 e maio de 2017. Não houve recusas dos gestores.

As entrevistas realizadas obedeceram a roteiro semiestruturado previamente testado, organizado em tópicos baseados nas categorias de análise para a regionalização da VS: planejamento/programação das ações; estratégias integradas de atuação para o enfrentamento de situações que exigem o compartilhamento da gestão; recursos financeiros e serviços de interesse disponibilizados no nível regional; integração das ações de VS e atenção básica em nível regional; papel da instância estadual; papel da Comissão Intergestores Regional (CIR); papel da VS na condução da rede de atenção à saúde na região. Os entrevistados assinaram um Termo de Consentimento Livre e Esclarecido (TCLE), sendo garantidos o sigilo e o anonimato dos envolvidos. Todas as entrevistas foram gravadas e transcritas.

Utilizou-se a análise temática de conteúdo com a codificação da informação nas categorias supracitadas. Para dar sentido ao material estudado, a análise envolveu uma etapa de pré-análise, abrangendo a escolha do material, a formulação de hipóteses, objetivos e a elaboração de indicadores para a interpretação dos resultados; a análise do material, que consistiu na codificação, categorização e quantificação da informação; e o tratamento dos resultados, que envolveu procedimentos diversos. Após a leitura das transcrições, foram identificados os conteúdos mais relevantes por meio da observação das convergências e divergências nas narrativas relacionadas com as categorias analíticas descritas. Os dados 
relevantes foram inseridos e ordenados em uma matriz de análise ${ }^{23,24}$. Buscou-se identificar os sentidos atribuídos pelos sujeitos às questões levantadas para entender a lógica interna desse grupo, em diálogo comparativo com a literatura.

A pesquisa cumpre os princípios éticos contidos na Declaração de Helsinki, tendo sido aprovada pelo Comitê de Ética em Pesquisa em seres humanos da Faculdade de Medicina da Universidade de São Paulo (FMUSP) com o número de protocolo 071/2015.

\section{Resultados e discussão}

A partir da percepção dos gestores estaduais da VS entrevistados, protagonistas do processo de regionalização na região de saúde, foi elaborada a matriz abaixo (quadro 1).

Quadro 1. Convergências e divergências temáticas referentes ao processo de regionalização da Vigilância em Saúde (VS), segundo a percepção de gestores estaduais da VS

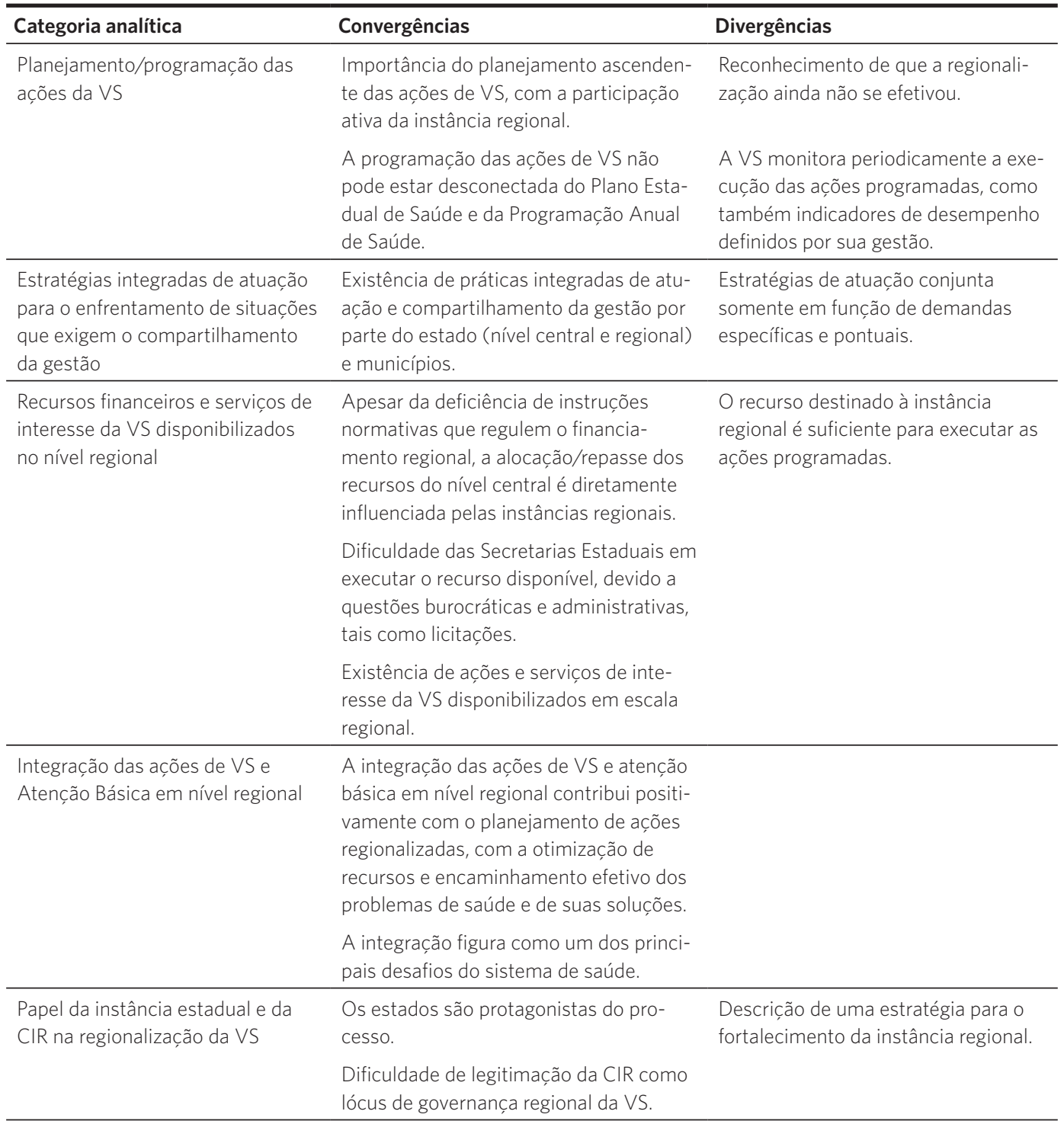


Quadro 1. (cont.)

\begin{tabular}{lll}
\hline Categoria analítica & Convergências & Divergências \\
\hline Papel da VS na condução da rede & Para que a integração da VS às redes de & \\
de atenção à saúde na região & atenção à saúde seja efetiva, é neces- \\
& sário que a indução aconteça no nível \\
& estratégico da gestão. \\
& Os gestores da VS reconhecem a neces- \\
& sidade de que a inserção e a atuação da \\
& vigilância sejam transversais à discussão \\
& das redes de atenção. \\
& Porém, esse reconhecimento ainda não \\
& é uma realidade por parte dos demais \\
& atores do sistema de saúde, a exemplo \\
& da atenção à saúde. \\
\hline
\end{tabular}

Fonte: Elaboração própria.

\section{Planejamento/programação das ações da VS em nível regional}

Os gestores reconheceram a importância do planejamento ascendente das ações de VS, com a participação ativa da instância regional, que é 'o braço no estado mais próximo dos municípios’, e apontaram que essas ações não podem estar desconectadas do Plano Estadual de Saúde e da Programação Anual de Saúde, os quais retratam a política de saúde e o planejamento das ações no estado. No entanto, em um estado que enfrenta dificuldades no processo de planejamento/programação com a inserção do nível regional, um gestor comenta:

Nunca tivemos uma divisão em regionais totalmente efetiva. Até pelas próprias características do estado. Nós temos o estado, digamos, de dimensões continentais e municípios idem. E isso, consequentemente, dificulta muito esse processo. [...] a gente tem que trabalhar muito com foco na realidade de cada município que compõe essas regionais. Até porque a regional aqui no estado ela funciona mais como uma questão de referência para a atenção à saúde. (Gestor 5).
Esse relato corrobora os achados de Viana e Lima25, ao investigar a regionalização nos estados brasileiros no período de 2007 a 2010. O estudo mostrou que a implementação da regionalização é dependente do contexto estadual e das regras mais gerais que conformam a política, sendo influenciado pela direção que se deseja imprimir ao processo.

Apesar do reconhecimento acerca da importância do planejamento das ações de VS por parte da maioria dos gestores entrevistados, apenas um relatou que a VS em seu estado monitora periodicamente a execução das ações programadas, como também indicadores de desempenho definidos pela gestão.

\footnotetext{
A gente monitora a cada reunião de colegiado [...] uma parte dessas prioridades. De modo que, pelo menos uma vez por mês, cada prioridade dessa seja monitorada a partir do que foi planejado. Também aqui a gente faz um monitoramento de indicadores de desempenho da gestão de Vigilância em Saúde. (Gestor 2).
}

A trajetória de desenvolvimento da VS no SUS sugere caminhos para uma avaliação sistemática de seu desempenho $0^{\mathbf{4 4}, 17,26}$ e para iniciativas locais de monitoramento qualificado 
capazes de melhorar a performance da VS27. Assim, o processo avaliativo da vigilância nos territórios deve construir pontes teórico-práticas entre as abordagens e a metodologia utilizada, ponderando sobre sua coerência e efetividade em relação aos princípios do SUS e das políticas e agendas promotoras da saúde, com a valorização do empoderamento local ${ }^{28}$.

\section{Estratégias integradas de atuação para o enfrentamento de situações que exigem o compartilhamento da gestão}

Outro núcleo de significação que emerge na fala dos gestores refere-se à prática integrada de atuação e compartilhamento da gestão por parte do estado (nível central e regional) e municípios. Os entrevistados apontaram a existência de grupo técnico (Gestor 1) e de reuniões mensais (Gestores 2 e 4) como estratégia para viabilizar a atuação compartilhada. O Gestor 2 exemplificou essa prática após a identificação da necessidade nas reuniões:

Um exemplo é [...] o controle do vetor do Aedes aegypti. É uma ação que é feita em parceria. $O$ UBV pesado, por exemplo, fica com o nível central. O nível central é que avalia o município e vê se ele tem condições de receber o UBV pesado [...] junto com a Geres [Gerência Regional de Saúde] [...] O inseticida das bombas costais fica na Geres, então o município precisando, é a Geres quem se responsabiliza para ir. O controle mais da visita do agente de endemias, aí é com o município. Ele é quem vai, quem coloca o larvicida, então é uma ação compartilhada entre os três níveis de gestão. (Gestor 2).

Apesar disso, os outros gestores afirmaram que, nos seus estados, a discussão de estratégias conjuntas acontece para demandas específicas e pontuais, em função de situações momentâneas, como descrito pelo Gestor 6: 'Então às vezes o 'fogo' faz com que a gente se integre, mas às vezes faz com que a gente se distancie".
Entende-se que a regionalização cooperativa conseguirá resolver alguns problemas gerados pela descentralização autárquica (unilateral, sem cooperação entre os entes) ${ }^{29}$, ao criar ações sinérgicas entre os gestores com maior compartilhamento de responsabilidades, ainda que, na prática, as questões relativas ao financiamento do sistema e à infraestrutura da rede de serviços sejam prioritárias na agenda da gestão, constituindo-se em um entrave ao pleno estabelecimento do processo de regionalização $0^{\mathbf{3} 30,31}$.

\section{Recursos financeiros e serviços de interesse da VS disponibilizados em nível regional}

Um dos desafios para o avanço do processo de regionalização da VS é a ausência de instruções normativas que regulem o financiamento regional ${ }^{\mathbf{1 4}}$. Os gestores expressaram que, apesar dessa deficiência, a alocação/ repasse dos recursos do nível central é diretamente influenciada pelas instâncias regionais.

No planejamento, que é onde se define e que norteia a aplicação do recurso, as prioridades e a alocação do recurso, onde vai ser o maior número de investimento, a gente não tem a menor dúvida que eles influenciam porque eles é que vão ajudar a Secretaria a definir onde está a maior carência, onde a vigilância precisa mais de sustentação financeira. (Gestor 4).

Quando questionados sobre a suficiência desses recursos, o posicionamento dos gestores foi divergente, destacando como dificuldade maior a execução financeira do recurso por parte das secretarias estaduais, devido a questões burocráticas e administrativas, a exemplo das licitações.

A existência de ações e serviços de interesse da VS disponibilizados em escala regional pode ser vista como indutora da regionalização ${ }^{32}$. A presença de Centros de Referência em Saúde do Trabalhador (Cerest) (Gestor 
1 e 6), de laboratórios regionais (Gestor 1, 2 e 6), de serviços de saúde com capacidade de realização de procedimentos de alta e média complexidade para doenças e agravos de interesse à VS (Gestor 2 e 6), de câmaras frias para armazenamento de imunobiológicos (Gestor 1,2 e 4) e de centrais de armazenamento e distribuição de equipamentos e inseticidas (Gestor 2 e 4 ) pode potencializar o processo de regionalização da VS.

Entretanto, alguns gestores (Gestor $1 \mathrm{e}$ 5) discordam dessa necessidade, apontando para maior responsabilidade do nível municipal no provimento desses serviços: "Como, a princípio, as ações na Vigilância em Saúde são descentralizadas, os municípios deveriam dar conta das suas ações” (Gestor 1).

Apesar disso, todos os gestores concordaram que a oferta de ações e serviços em escala regional contribui para a integralidade e para a ampliação do acesso ao sistema de saúde. Ao se reconhecer que municipalização não foi capaz de proporcionar a integração de serviços, instituições e práticas nos territórios, nem a formação de arranjos mais cooperativos na saúde ${ }^{33}$, a regionalização passou a ser priorizada como macroestratégia organizativa do sistema de saúde brasileiro ${ }^{14}$.

Não tem outro jeito. Essa é a única forma que a gente vai poder realmente chegar mais próximo e estar junto e conseguir resolver os problemas de uma forma muito mais coerente. [...] Imagine, eu tenho uma equipe aqui [...] ainda [...] fazendo execução [...] o tamanho que nós temos, a diferença geográfica que a gente tem, ter que sair daqui pra executar, planejar e fazer tudo pro estado, a equipe aqui? (Gestor 6).

Assim, para que a regionalização de ações e serviços de saúde seja consolidada, o desenvolvimento das relações intergovernamentais deve ser considerado, observando a inserção de figuras regionais como instâncias administrativas não coincidentes com os entes federados ${ }^{3}$.

\section{Integração das ações de VS e atenção básica em nível regional}

Os entrevistados enfatizaram que a integração das ações de VS e atenção básica em nível regional contribui positivamente para o planejamento de ações regionalizadas, para a otimização de recursos e encaminhamento efetivo dos problemas de saúde e de suas soluções. Porém, percebe-se, nas falas, que essa integração ainda figura como um dos principais desafios do SUS ${ }^{34,35}$.

Não dá pra pensar, por exemplo, a estratégia saúde da família sem as ações de vigilância. Porque existe muito ainda essa fala de que as ações de vigilância são executadas por pessoas que trabalham na vigilância e isso não é verdade. Vigilância a gente tem desde a atenção primária a té o hospital. Então não dá mais pra ter esse pensamento. Então eu acho que essa integração era fundamental pra que muita coisa desse certo. Inclusive, que a gente conseguisse desafogar muito as emergências, que a gente conseguisse acabar com a superlotação dos hospitais. Eu acho que se essa integração se desse na ponta mesmo, onde tem que dar lá na atenção básica, eu acho que a gente conseguiria muitos avanços. (Gestor 2).

A ação integrada necessita ser planejada e executada de forma horizontal, aglutinando o saber dos sujeitos considerando as singularidades da prática de cada um, na perspectiva de construir propostas e ações coerentes com as necessidades da população $0^{34}$.

\section{Papel da instância estadual e da CIR na regionalização da VS}

Os discursos apontaram para os estados como protagonistas do processo de regionalização da VS.

[...] é o condutor da política de regionalização.

[...] Ele que deflagra, ele que articula, ele que [...] prepara todo o arcabouço legal para que isso aconteça. Que aconteça todo processo de regionalização 
em todas as instâncias. Ele que envolve os municípios. Se o estado não se movimentar pra isso, a regionalização não acontece. Ele é o indutor. (Gestor 4).

Apesar de reconhecer a importância do âmbito estadual, apenas um entrevistado mencionou a existência de uma estratégia para o fortalecimento da instância regional, a qual contemplava ações de estruturação, a exemplo da contratação de apoiadores institucionais, repasse regular de recursos financeiros e aquisição de veículos; e de qualificação, por meio de um plano de formação continuada dos profissionais a partir das necessidades locais.

Um estudo realizado por Vargas et al.36, ao analisar a regionalização no Brasil, concluiu que esse processo apresenta baixa implantação e desenvolvimento, pois depende de responsabilidades complexas da entidade regional e da fragilizada corresponsabilização dos níveis estadual e federal. Isso porque o processo de regionalização vem se associando, em cada estado, à dinâmica socioeconômica, às políticas de saúde anteriores, ao grau de articulação existente entre os diferentes atores e sua capacidade de gerar consensos sobre a divisão de responsabilidades gestoras e desenhos regionais adotados ${ }^{37}$. Entende-se, portanto, que a capacidade de articulação do gestor estadual em promover o envolvimento dos atores e gerar o sentimento de pertencimento regional é uma tarefa complexa, mas crucial para o desenvolvimento de uma regionalização cooperativa ${ }^{31}$.

No que se refere à CIR, é notório que existe uma fragilidade na participação da VS, com presença esporádica e geralmente voltada para as ações de controle de vetores ou discussão de repasses e recursos financeiros. Essa instância de pactuação e cogestão ainda encontra dificuldade de ser legitimada como lócus de governança regional da VS. Ao propor essa problematização, o intuito não é anular o papel da CIR no processo de regionalização da VS, mas destacar que essa fragilidade pode comprometer o fortalecimento da VS nesse âmbito de atuação regional. $\mathrm{O}$ aprendizado institucional fruto da dinâmica de participação na CIR fortalece a gestão intergestores e se apresenta como importante componente para construção do processo de regionalização ${ }^{\mathbf{3 8}}$.

\section{Papel da VS na condução da rede de atenção à saúde na região}

Para o Gestor 1, o papel da VS é "trazer informação, dados. Subsidiar informação, ajudar o diagnóstico. E a outra seria a própria execução de algumas ações”. A VS, portanto, é responsável pela informação para a ação e por intervenções que reduzam riscos e promovam a saúde nos territórios, integrada às redes de atenção à saúde, um de seus principais desafios na atualidade. Assim, é imperativo que a vigilância se reconheça na agenda da determinação social da saúde, produzindo conhecimentos e práticas intersetoriais ${ }^{39}$.

Entretanto, segundo relato dos entrevistados, para que essa integração às redes seja efetiva, é preciso que a indução aconteça no nível estratégico da gestão.

Eu penso que a diretriz, a indução tem que partir do nível estratégico da Secretaria. Pra poder trabalhar e trazer a vigilância para junto das redes. Para fazer parte, de alguma maneira, das redes. Porque a vigilância, por si só, não consegue 'startar' um processo dessa natureza. Agora, ela pode entrar como partícipe do processo. (Gestor 4).

Um avanço pode ser percebido à medida que os gestores da VS reconhecem a necessidade de que a inserção e a atuação da vigilância sejam transversais à discussão das redes de atenção, ainda que os envolvidos na atenção nem sempre considerem assim. Isso pode se dever ao fato de que o contradiscurso dos profissionais da assistência seja mais forte em relação aos da vigilância.

Pra gente que faz a vigilância é muito claro a importância da vigilância ser transversal a toda essa discussão das redes. Mas é muito interessante 
como na prática quem é da atenção à saúde não consegue captar muito e ver essa [...] importância! [...] Como é que cabe? Como é que vai caber a vigilância nessa discussão de redes se a gente tá falando em maternidade. [...] De onde é que a gente vai ter maternidade no estado, onde é que não vai ter? Onde é que vale a pena ter? Como é que vai se constituir essa rede? (Gestor 2).

A publicação da Política Nacional de Vigilância em Saúde (PNVS), por meio da Resolução $n^{\circ} 588$, de 12 de julho de 2018 , aponta para necessidade de inserção das ações de VS em todas as instâncias e pontos da rede de atenção do SUS, mediante articulação e construção conjunta de protocolos, linhas de cuidado e matriciamento da saúde, bem como na definição das estratégias e dispositivos de organização e fluxos da rede de atenção ${ }^{40}$.

Essa política destaca como estratégias para organização da VS a regionalização das ações e serviços articulados com a atenção em saúde no âmbito da região de saúde; processos de trabalho integrados com a atenção à saúde, que devem considerar o planejamento integrado da atenção como ferramenta para a definição de prioridades comuns para atuação conjunta; e a inserção da VS na rede de atenção, que deve contribuir para a identificação de riscos e situações de vulnerabilidade. Com isso, espera-se que a articulação dos saberes, processos e práticas relacionados com a VS alinhe-se com o conjunto de políticas de saúde no âmbito do SUS, considerando a transversalidade de suas ações sobre a determinação do processo saúde-doença ${ }^{40}$.

\section{Considerações finais}

O estudo, apesar de restrito à percepção de gestores estaduais, traz contribuições relevantes, pois permitiu identificar que o processo de implementação da regionalização da VS no Brasil acontece de forma assimétrica e é diretamente influenciado pelo sistema organizacional no qual se insere. Por meio das entrevistas, foi possível identificar convergências e divergências de concepções acerca das categorias de análise utilizadas, o que reafirma o tensionamento entre o que está instituído nas bases normativas e o que é operacionalizado no nível local.

Em que pese a regulação da esfera federal por meio de normas e portarias, o processo instituído de regionalização da VS esbarra em contextos locais, cujo enfoque operacional ainda é marcado pela busca de soluções municipais para problemas regionais. Ademais, mesmo com os avanços, a exemplo da publicação da PNVS, e os esforços dos gestores do Sistema Nacional de Vigilância em Saúde (SNVS), a regionalização ainda não se encontra efetivamente na agenda prioritária da instância estadual, a não ser no que se refere à organização assistencial dos serviços.

A abordagem utilizada demonstrou que, se, por um lado, os estados figuram como protagonistas do processo de regionalização da VS, por outro, a existência de estratégias e mecanismos para o fortalecimento do nível regional ainda é escassa. Ressalta-se que o aprimoramento de processos de planejamento ascendente e de práticas de monitoramento e avaliação com a participação ativa dos atores regionais pode apontar um caminho para a regionalização cooperativa, uma vez que o âmbito regional não é um novo ente político, mas dependente da capacidade e da solidariedade das três esferas de governo.

Ressalta-se que, apesar das narrativas não fazerem referência alguma aos objetos e práticas das vigilâncias sanitária e de saúde do trabalhador, todos os entrevistados eram gestores de VS, e, portanto, também responsáveis pelas ações das referidas vigilâncias. Além disso, o instrumento de pesquisa abordou de forma explícita o conceito de VS adotado pelo estudo, pautado na Portaria $n^{\circ} 1.378$, de 9 de junho de $2013^{13}$. Sabe-se que esse conceito foi sendo ressignificado e que o lançamento da PNVS reflete o esforço e os debates, nem sempre consensuais, em torno do seu escopo de atuação ${ }^{\mathbf{4 1}}$. No entanto, é possível perceber uma certa 
fragmentação institucional das ações de VS, organizadas em bases de conhecimentos e de práticas por vezes independentes, o que desperdiça um enorme potencial analítico e de intervenção sobre os condicionantes do processo saúde-doença.

A apropriação de espaços de pactuação e cogestão, como é o caso da CIR, ainda é um desafio para consolidação da regionalização da VS, especialmente pela pouca participação da vigilância nas pautas de discussão, frequentemente permeada por questões referentes à oferta insuficiente de serviços, majoração dos salários e procedimentos (particularmente de médicos); superlotação em hospitais; Estratégia Saúde da Família pouco resolutiva, entre outros ${ }^{37}$. Contudo, é necessário não perder de vista a compreensão de que a CIR representa um mecanismo potente para legitimar relações de corresponsabilidade.

Identifica-se, na fala dos gestores, um discurso coerente com os resultados de pesquisas que investigaram a integração das ações de VS e atenção básica no nível local ${ }^{34,35}$. O reconhecimento de que essa integração propicia uma maior efetividade na solução dos problemas de saúde e a otimização de recursos acompanha a constatação de que esse é um dos principais desafios do sistema. Concomitantemente, destaca-se o importante papel da VS na identificação de riscos e situações de vulnerabilidade, uma vez inserida na rede de atenção à saúde. Todavia, a dificuldade em reconhecer o lugar de atuação da vigilância nessa discussão de redes também é apontada pelos entrevistados como um desafio a ser superado.

A inexistência de normativas que regulem o financiamento regional pode ser considerada um gargalo para o avanço do processo de regionalização da VS. Não obstante, a efetivação da regionalização pressupõe laços cooperativos interinstitucionais e compromisso político que transcendam o atendimento a preceitos legais e normativos. Em síntese, isso exigirá um esforço no sentido de obter uma maior precisão quanto ao papel de cada ente federativo na organização do SNVS regionalizado, particularmente o papel do estado no direcionamento do processo de regionalização e na identificação dos entraves que impedem sua consolidação.

Ainda que as convergências identificadas nas falas dos gestores entrevistados apontem para um caminho de implementação da regionalização da VS, as contradições entre o legitimado como desejável e o instituído como prática representam limites concretos de efetivação da regionalização, não só no sentido político-institucional, mas também definidas pelos sentidos que os agentes verbalizam e reproduzem em práticas de saúde.

\section{Colaboradores}

Albuquerque AC (0000-0001-6305-4127)* realizou a coleta e análise dos dados, participou da concepção e planejamento do estudo, redação do manuscrito e aprovação da versão final do artigo. Cesse EAP (0000-0001-57453981)*, Samico IC (0000-0002-8338-7946)*, Felisberto E (0000-0002-2316-2251)* e Frias PG (0000-0003-4497-8898)* participaram da concepção e planejamento do estudo e colaboraram na redação, revisão crítica e aprovação da versão final do artigo. Silva GC (0000-0002-5247-8386)* realizou a coleta e análise dos dados, participou da revisão crítica e aprovação da versão final do artigo. 


\section{Referências}

1. Brasil. Lei no 8.080, de 19 de setembro de 1990. Dispõe sobre as condições para a promoção, proteção e recuperação da saúde, a organização e o funcionamento dos serviços correspondentes e dá outras providências. Diário Oficial da União. 20 Set 1990.

2. Paim JS, Teixeira CF. Configuração institucional e gestão do Sistema Único de Saúde: problemas e desafios. Ciênc. Saúde Colet. 2007; 12(supl):1819-1829.

3. Dourado DA, Elias PEM. Regionalização e dinâmica política do federalismo sanitário brasileiro. Rev. Saúde Pública. 2011; 45(1):204-211.

4. Arretche M. Federalismo e relações intergovernamentais no Brasil: a reforma de programas sociais. Dados. 2002; 45(3):431-458.

5. Viana ALA, Machado CV. Descentralização e coordenação federativa: a experiência brasileira na saúde. Ciênc. Saúde Colet. 2009; 14(3):807-817.

6. Machado CV. O modelo de intervenção do Ministério da Saúde nos anos 90. Cad. Saúde Pública. 2007; 23(9):2113-2126.

7. Viana ALd'A, Heimann LS, Lima LD, et al. Descentralização no SUS: efeitos da NOB-SUS 01/96. In: Negri B, Viana ALd'A, organizadores. O Sistema Único de Saúde em dez anos de desafio. São Paulo: Sobravime; 2002. p. 471-488.

8. Campos GWS. Efeitos Paradoxais da Descentralização no Sistema Único de Saúde do Brasil. In: Fleury S, organizadora. Democracia, Descentralização e Desenvolvimento: Brasil e Espanha. Rio de Janeiro: FGV; 2006. p. 417-442.

9. Santos L, Andrade LOM. Redes interfederativas de saúde: um desafio para o SUS nos seus vinte anos. Ciênc. Saúde Colet. 2011; 16(3):1671-1680.

10. Lima LD. A coordenação federativa do sistema público de saúde no Brasil. In: Noronha JC, Pereira TR, organizadores. A saúde no Brasil em 2030 - prospec- ção estratégica do sistema de saúde brasileiro: organização e gestão do sistema de saúde. Rio de Janeiro: Fiocruz/Ipea/Ministério da Saúde/Secretaria de Assuntos Estratégicos da Presidência da República; 2013. p. 73-139.

11. Brasil. Ministério da Saúde. Comissão Intergestores Tripartite. Resolução $n^{\circ} 23$, de 17 de agosto de 2017. Estabelece diretrizes para o processo de Regionalização, Planejamento Regional Integrado, elaborado de forma ascendente, e Governança das Redes de Atenção à Saúde no âmbito do SUS. Diário Oficial da União. 18 Ago 2017.

12. Brasil. Ministério da Saúde. Comissão Intergestores Tripartite. Resolução n ${ }^{\circ}$ 37, de 22 de março de 2018. Dispõe sobre o processo de Planejamento Regional Integrado e a organização de macrorregiões de saúde. Diário Oficial da União. 26 Mar 2018.

13. Brasil. Ministério da Saúde. Portaria $\mathrm{n}^{0} 1.378$, de 9 de julho de 2013. Regulamenta as responsabilidades e define diretrizes para execução e financiamento das ações de Vigilância em Saúde pela União, Estados, Distrito Federal e Municípios, relativos ao Sistema Nacional de Vigilância em Saúde e Sistema Nacional de Vigilância Sanitária. Diário Oficial da União. 10 Jul 2013.

14. Albuquerque AC, Cesse EAP, Felisberto E, et al. Regionalização da vigilância em saúde: uma proposta de avaliação de desempenho em uma região de saúde no Brasil. Rev Bras Saude Mater Infant. 2017; 17(supl1):135-151.

15. Oliveira CM, Cruz MM. Sistema de Vigilância em Saúde no Brasil: avanços e desafios. Saúde debate. 2015; 39(104):255-267.

16. Lins AM, Cecilio LCO. O discurso da institucionalização de práticas em saúde: uma reflexão à luz dos referenciais teóricos das ciências humanas. Physis. 2008; 18(3):483-499.

17. Silva Júnior JB. Epidemiologia em serviço: uma avaliação de desempenho do Sistema Nacional de Vigi- 
lância em Saúde [tese]. Campinas: Faculdade de Ciências Médicas da Universidade Estadual de Campinas; 2004.

18. Brasil. Ministério da Saúde; Fundação Nacional de Saúde. Projeto VIGISUS - Estruturação do Sistema Nacional de Vigilância em Saúde. Brasília, DF: MS; 1998.

19. Brasil. Ministério da Saúde, Secretaria de Vigilância em Saúde. Portaria no 1.708, de 16 de agosto de 2013. Regulamenta o Programa de Qualificação das Ações de Vigilância em Saúde (PQAVS), com a definição de suas diretrizes, financiamento, metodologia de adesão e critérios de avaliação dos Estados, Distrito Federal e Municípios. Diário Oficial da União. 16 Ago 2013.

20. Carvalho ALB, Jesus WLA, Senra IMVB. Regionalização no SUS: processo de implementação, desafios e perspectivas na visão crítica de gestores do sistema. Ciênc. Saúde Colet. 2017; 22(4):1155-1164.

21. Viana ALd'A, Ferreira MP, Cutrim MA, et al. O Processo de Regionalização no Brasil: influência das dimensões Política, Estrutura e Organização. Rev Bras Saude Mater Infant. 2017; 17(supl1):27-43.

22. Viana ALd'A, Bousquat A, Pereira APCM, et al. Tipologia das regiões de saúde: condicionantes estruturais para a regionalização no Brasil. Saude soc. 2015; 24(2):413-422.

23. Minayo MCS, organizadora. O desafio do conhecimento: pesquisa qualitativa em saúde. São Paulo: Hucitec; 2010.

24. Bardin L. Análise de conteúdo. São Paulo: Edições $70 ; 2011$.

25. Viana ALd'A, Lima LD, organizadoras. Regionalização e relações federativas na política de saúde do Brasil. Rio de Janeiro: Contra-capa; 2011.

26. Costa JMBS, Cesse EAP, Samico IC, et al. Avaliação do desempenho estadual da vigilância em saúde de Pernambuco. Physis. 2015; 25(4):1141-1163.
27. Hartz Z. Do Monitoramento do Desempenho ao Desempenho do Monitoramento: Novas Oportunidades para Avaliação na Gestão da Vigilância em Saúde. Ciênc. Saúde Colet. 2013; 18(5):1201-1216.

28. Gallo E, Setti AFF. Abordagens ecossistêmica e comunicativa na implantação de Agendas territorializadas de desenvolvimento sustentável e promoção da saúde. Ciênc. Saúde Colet. 2012; 17(6):1433-1446.

29. Pestana M, Mendes EV. Pacto de gestão: da municipalização autárquica à regionalização cooperativa. Belo Horizonte: Secretaria do Estado de Saúde de Minas Gerais; 2004.

30. Fleury S, Ouverney ALM, Kronemberger TS, et al. Governança local no sistema descentralizado de saúde no Brasil. Rev. Panam Salud Publica. 2010; 28(6):446-455.

31. Lima LD, Viana ALd'A, Machado CV, et al. Regionalização e acesso à saúde nos estados brasileiros: condicionantes históricos e político-institucionais. Ciênc. Saúde Colet. 2012; 17(11):2881-2892.

32. Brasil. Ministério da Saúde, Secretaria de Vigilância em Saúde. Manual de gestão da Vigilância em Saúde. Brasília, DF; 2009.

33. Teixeira CF, Solla JP. Modelo de atenção à Saúde no SUS: trajetória do debate conceitual, situação atual, desafios e perspectivas. In: Lima NT, Gerschman S, Edler FC, et al., organizadores. Saúde e Democracia: história e perspectiva do SUS. Rio de Janeiro: Fiocruz; 2005. p. 451-477.

34. Santos SSBS, Melo CMM. Avaliação da descentralização da vigilância epidemiológica para a Equipe de Saúde da Família. Ciênc. Saúde Colet. 2008; 13(6):1923-1932.

35. Barbosa MCL, Costa MCN, Teixeira MG, et al. Efeitos da descentralização das ações de vigilância epidemiológica para as equipes de saúde da família. Epidemiol Serv Saúde. 2010; 19(4);347-354.

36. Vargas I, Mogollón-Pérez AS, Unger JP, et al. Regional-based Integrated Healthcare Network policy in 
Brazil: from formulation to practice. Health Policy Plan. 2014; 30(6):705-717.

37. Reis YAC, Cesse EAP, Carvalho EF. Consensos sobre o papel do gestor estadual na regionalização da assistência à saúde no Sistema Único de Saúde (SUS). Rev Bras Saude Mater Infant. 2010; 10(supl1):157-172.

38. Santos AM, Giovanella L. Governança regional: estratégias e disputas para gestão em saúde. Rev. Saúde Pública. 2014; 48(4):622-631.

39. Franco Neto G, Villardi JWR, Machado JMH, et al. Vigilância em Saúde brasileira: reflexões e contribuição ao debate da la Conferência Nacional de Vigilância em Saúde. Ciênc. Saúde Colet. 2017; 22(10):31373148.
40. Brasil. Conselho Nacional de Saúde. Resolução no 588, de 12 de julho de 2018. Institui a Política Nacional de Vigilância em Saúde (PNVS). Diário Oficial da União. 12 Jul 2018.

41. Teixeira MG, Costa MCN, Carmo EH, et al. Vigilância em Saúde no SUS - construção, efeitos e perspectivas. Ciênc. Saúde Colet. 2018; (23):1811-8.

\footnotetext{
Recebido em 02/12/2019

Aprovado em 08/10/2020

Conflito de interesses: inexistente

Suporte financeiro: não houve
} 